\title{
Prevalent placement error of deep brain stimulation electrode in movement disorders (technical considerations)
}

\author{
Kloc $\mathrm{M}^{1}$, Kosutzka $\mathrm{Z}^{2}$, Steno $\mathrm{J}^{1}$, Valkovic $\mathrm{P}^{2,3}$ \\ Department of Neurological Surgery, Faculty of Medicine, Comenius University and University Hospital \\ in Bratislava, Bratislava, Slovakia.klocmichal@gmail.com
}

\begin{abstract}
BACKGROUND: Deep brain stimulation is an effective and safe technique. Displacement of the electrode relative to the optimal stimulation site can lead to insufficient effect and sometimes to the need of operative electrode re-position.

OBJECTIVE: This study was aimed to analyse targeting accuracy of deep brain stimulation electrode implantation to subthalamic nucleus (STN) and globus pallidus internus (Gpi). It detected possible causes of inaccuracy and prevalent shift to certain direction.

METHODS: Targeting accuracy was analysed in 47 patients with Parkinson's disease (PD) and 11 patients with dystonia with bilateral implantation of deep brain stimulation electrodes between years 2009 and 2016. RESULTS: A shift of electrode to prevalent direction was observed on the left side to medial and posterior and on the right side to lateral direction. Greater shift was observed on the left side and in a higher angulation of trajectory laterally. Movement of the electrode, because of its traction in anchoring device, was identified as a possible factor for prevalent electrode shift. Calibration of stereotactic coordinates to correct prevalent shift was used. CONCLUSION: Targeting inaccuracy is the result of accumulation of errors in individual steps of electrode implantation. Direction of the shift can be random or it can be toward a prevalent direction. A correction of prevalent error can prevent a suboptimal electrode placement (Tab. 3, Fig. 11, Ref. 29). Text in PDF www.elis.sk. KEY WORDS: deep brain stimulation, calibration, targeting accuracy, stereotactic techniques.
\end{abstract}

\section{Introduction}

Deep brain stimulation is used for treatment of extrapyramidal movement disorders, epilepsy, psychiatric disorders and chronic pain. Because of its effectivity in treatment and reversibility, it became popular and wide spread.

The key feature of deep brain stimulation electrode implantation is the accuracy of targeting toward the optimal site of stimulation and safety for the patients.

An exact algorithm of operative procedure depends on decisions and experience of the operation team including neurosurgeon, neurologist, radiologist and other cooperating participants of the treatment.

The target structure of the stimulation is often small, optimal effect depends on the accurate targeting with the least error. Suboptimal placement of the electrode more than $2 \mathrm{~mm}$ away can lead to suboptimal effect of the stimulation $(1,2,3)$.

${ }^{1}$ Department of Neurological Surgery Faculty of Medicine of Comenius University and University Hospital in Bratislava, Bratislava, Slovakia, ${ }^{2}$ IInd Department of Neurology Faculty of Medicine of Comenius University and University Hospital in Bratislava, Bratislava, Slovakia, and ${ }^{3}$ Institute of Normal and Pathological Physiology, Slovak Academy of Sciences, Bratislava, Slovakia

Address for correspondence: M. Kloc, MD, Department of Neurological Surgery Faculty of Medicine of Comenius University and University Hospital in Bratislava, Limbova 5, SK-833 05 Bratislava, Slovakia. Phone: +421.2.59542532, Fax: +421.2.59543532
That is the reason why three-dimensional mapping of the target is used with up to five electrodes, one in the middle and four to each direction around the central track. Microelectrode recording (MER) and intraoperative stimulation is used in order to tackle the target properly. Simultaneous use of five microelectrodes improves the probability to reveal ideal position of the target. However, this also has a higher risk of haemorrhagic complications, which could have devastating impact for the patient. With the advances in imaging, registration and targeting, magnetic resonance (MR)-guided technique enables to use less tracks with a preserving accuracy.

The aim of this study was to analyse targeting accuracy using five tracks in order to collect sufficient information. By this analysis, we tried to support the concept of reducing the number of microelectrode tracks used. The results suggested that there was a targeting error to prevalent direction and therefore we compared the electrode position relative to the planned trajectory.

\section{Methods}

We analysed 47 patients with idiopathic Parkinson's disease (PD) and 11 patients with dystonic syndrome, who had undergone bilateral implantation of DBS electrodes between years 2009 and 2016. All patients had surgery in one centre and all operations were performed by one neurosurgeon (M.K.).

All patients were referred from tertiary movement disorder centre and the indication for DBS kept the standard protocol by multidisciplinary committee (4). 


\section{7-653}

All patients with dystonia were bilaterally implanted into Gpi and patients with Parkinson's disease to STN.

Stereotactic system Leksell was used. Stereotactic CT was merged with 3T MR. In all patients, three-dimensional T1 MR with double dose contrast agent was performed, other MR protocols were used according to specific target structure, in STNthree-dimensional T2 $1 \mathrm{~mm}$ or $2 \mathrm{~mm}$ slice, in Gpi-proton density (PD), Flair.

Targeting in FrameLink used a direct target visualisation, relation to adjacent anatomical structures, stereotactic atlas WahrenSchaltenbrand and statistic distance of the target from the middle of AC-PC. Reconstruction of images and merging with registration was automatic in FrameLink. The accuracy of merging was checked carefully in all planes, in some cases it was corrected manually by setting corresponding points - „landmarks“. Trajectory was planned bilateral in front of the coronar suture avoiding sulci and ventricles.

Operation was performed under a local anesthesia in patients with PD and under a general anesthesia or sedation and analgesia in dystonia subjects with flattening of anesthesia during microelectrode recording. All electrodes were anchored in the burr hole with the Medtronic StimLock burr hole cover.

MER and intraoperative stimulation were performed with five microelectrodes in 27 patients and with three microelectrodes in 20 patients with $\mathrm{PD}$ and 11 patients with dystonia.

Immediately after the electrode implantation in latter 31 patients (with three microelectrodes used), stereotactic CT was performed. This CT was used after its merging with preoperative CT and MR to evaluate the shift of the planned trajectory relative to the final electrode position. The lead placement verification using postoperative MR and CT showed an equivalent lead representation (5).

\section{Results}

In 27 consecutive patients with $\mathrm{PD}$, deep brain stimulation electrodes were implanted bilaterally ( 54 electrodes) to STN. Five microelectrode tracks were used for three-dimensional mapping of the target area. Significant MER activity in more than $1 \mathrm{~mm}$ length of the trajectory was found in the following numbers of microelectrodes:

1 electrode $-1.8 \%(1)$

2 electrodes $-9.3 \%(5)$

3 electrodes $-22.2 \%$ (12)

4 electrodes $-22.2 \%$ (12)

5 electrodes $-44.4 \%$ (24)

In $44.4 \%$, significant MER activity was found in all five tracks and in almost $90 \%$ it was present in three or more tracks.

Intraoperative stimulation was not tested in all five electrodes. If there was a typical MER activity in the central electrode, this electrode was tested supposedly having the best location relative to the optimal site for the stimulation. Another electrode was tested if there was more typical MER activity in other track compared to the central electrode or if there was not sufficient effect of the

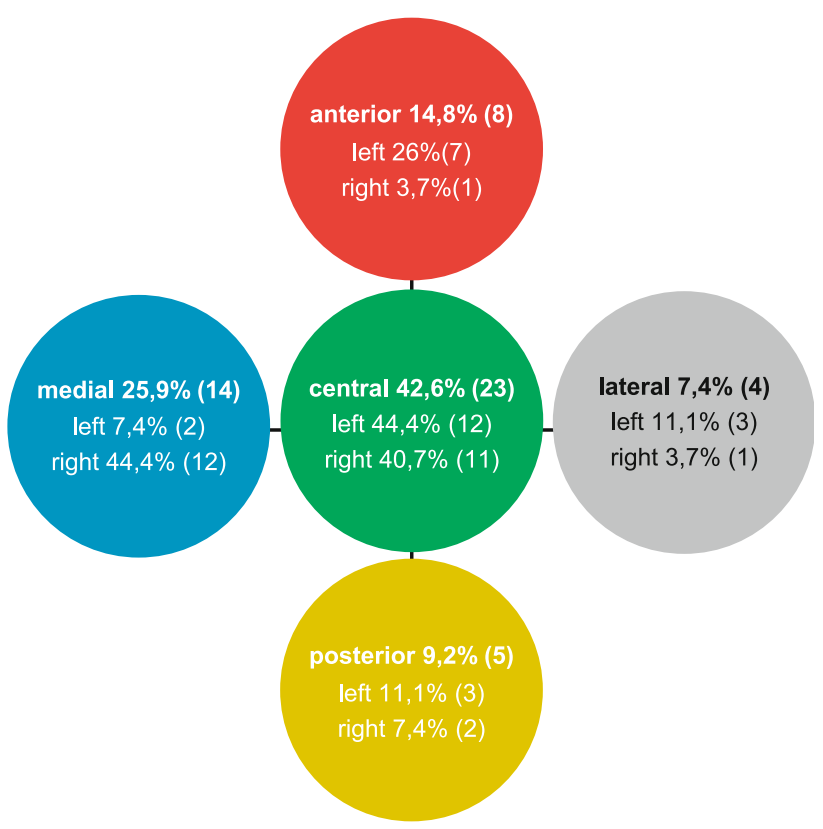

Fig. 1. Number of penetrations used for definitive electrode implantation.

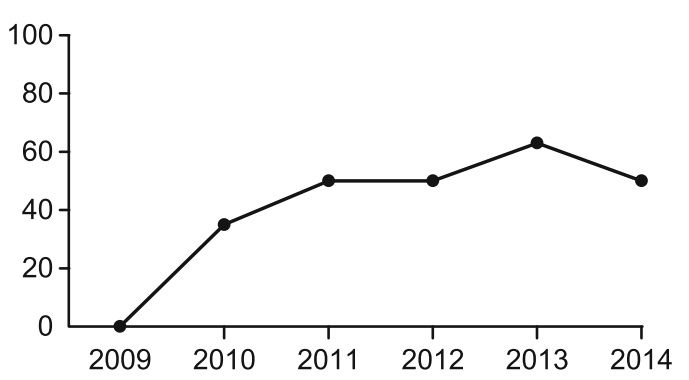

Fig. 2. Portion of definitive electrodes implanted to central penetration in \% in individual years.

stimulation on motor signs of PD or if there were some adverse effects during the stimulation in the central track. Certain adverse stimulation effects indicated the direction to the optimal site of the stimulation according to which anatomical structure was affected by the stimulation. Typically, 2 electrodes were tested in intraoperative stimulation.

If effect of the intraoperative stimulation was similar in more electrodes preferentially, the central track was used for definitive electrode implantation (Fig. 1).

Based on this data, we decided to reduce the number of tracks from five to three according to the most often used tracks for a definitive electrode placement on the left side to anterior-centrallateral and on the right side to central-medial-posterior. Objective for the use of less electrodes was to make operation safer and prevent haemorrhagic complications by a decreasing number of penetrations.

During the course of individual years, we observed the learning curve of electrode implantation to the central track (Fig. 2). 
The results of electrode placements suggested that there occurred a shift of the electrode position different for both sides of the brain. In the next consecutive 20 patients with PD (40 electrodes) and in 11 patients with dystonia (22 electrodes), three microelectrodes were used for 3D mapping of the target area. Immediately postoperatively, stereotactic CT was performed with implanted electrodes. In FrameLink, this CT was merged with preoperative imaging and we compared the definitive position of the electrode relative to the planned trajectory. Scalar error was counted from Heron's formula (Fig. 3):

$h=\frac{2}{c}(\sqrt{\mathrm{s}(s-a)(s-b)(s-c)})$

where $s=\frac{(a+b+c) s=(a+b+c)}{2}$

The scalar error was divided to vector errors in $\mathrm{x}, \mathrm{y}$ axis. The error in $\mathrm{z}$ axis is the least significant because it can be corrected without changing the trajectory, that is why we were interested mostly in the error in $\mathrm{x}$ and $\mathrm{y}$ axis. We evaluated this error separately for both sides of the brain.

In patients with PD with the target in STN, vector error in $\mathrm{x}$ axis was on the left side on average $1.065 \mathrm{~mm}$ to medial direction and in y axis $0.3 \mathrm{~mm}$ toward posterior direction. The standard deviation was 1.27 , more individual errors were close or more than 2 $\mathrm{mm}$, the maximal error to the medial direction was $3.6 \mathrm{~mm}$ (Fig. 4).

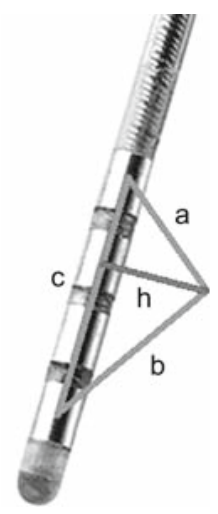

Fig. 3. Calculation of scalar error of electrode shift; $c$ is distance between centre of proximal and distant contact of the electrode.

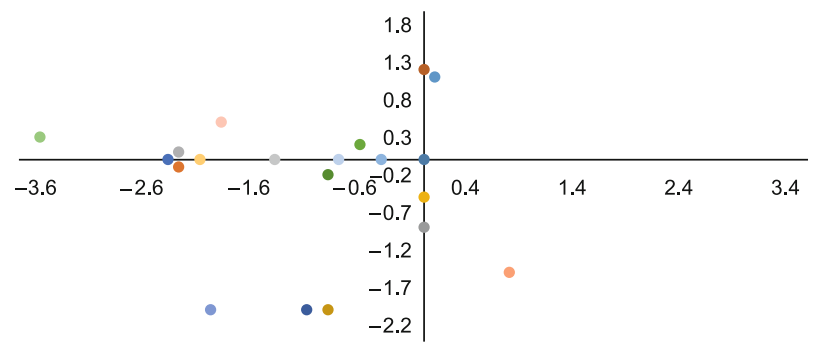

Fig. 4. Distribution of error in axial plane STN left side in $\mathbf{m m}$.

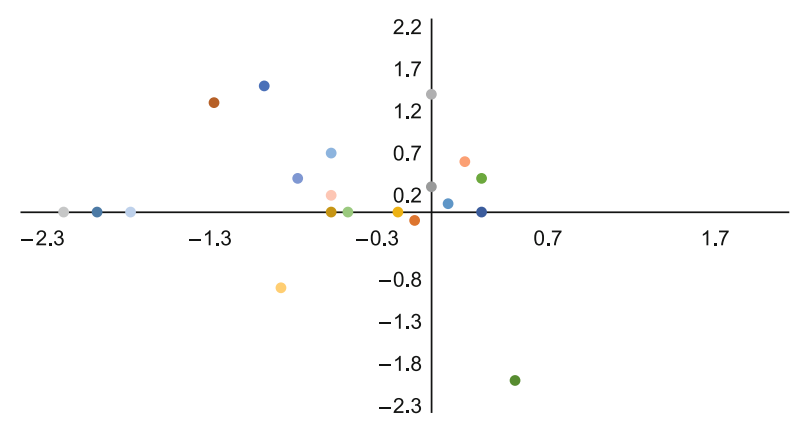

Fig. 5. Distribution of error in axial plane STN right side in $\mathbf{m m}$.

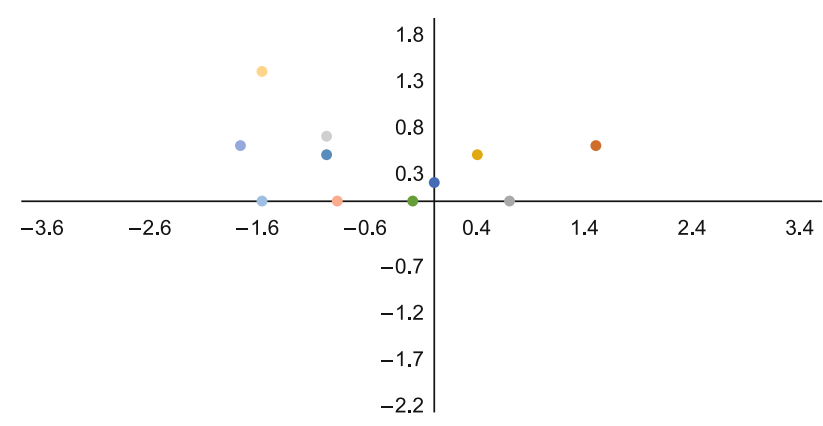

Fig. 6. Distribution of error in axial plane Gpi left side in $\mathbf{m m}$.

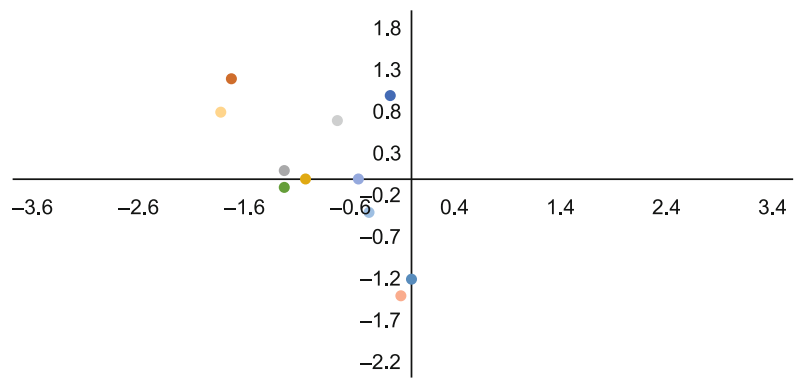

Fig. 7. Distribution of error in axial plane Gpi right side in $\mathbf{m m}$.

On the right side of the brain, vector error in $\mathrm{x}$ axis was 0.55 $\mathrm{mm}$ to the lateral direction and in $\mathrm{y}$ axis 0.24 to the anterior direction with the standard deviation 0.62 (Fig. 5).

In patients with dystonia with electrodes implanted to Gpi, vector error on the left side was $0.5 \mathrm{~mm}$ to the medial direction for $\mathrm{x}$ axis and $0.41 \mathrm{~mm}$ to the anterior direction in $\mathrm{y}$ axis (Fig. 6).

On the right side of the brain, there was vector error in $\mathrm{x}$ axis $0,8 \mathrm{~mm}$ to the lateral direction and $0,06 \mathrm{~mm}$ to the anterior direction in y axis (Fig. 7).

Distribution of error was in some cases proved to be prevalent to one direction- left STN in $\mathrm{x}$ axis to medial, right STN in $\mathrm{x}$ axis to lateral, right Gpi in $\mathrm{x}$ axis to the lateral direction. We tried to exclude any technical error and possible causes were analysed. Tool for error with the prevalent direction correction can be calibration of stereotactic coordinates (Tab. 1). 
Tab. 1. Recommended calibration of stereotactic coordinates.

\begin{tabular}{lcc}
\hline STN & Left & Right \\
\hline X coordinate correction & $+1 \mathrm{~mm}$ & $0.5 \mathrm{~mm}$ \\
Y coordinate correction & $+0.3 \mathrm{~mm}$ & 0 \\
\hline Gpi & Left & Right \\
\hline X coordinate correction & 0 & $+0.8 \mathrm{~mm}$ \\
Y coordinate correction & 0 & 0 \\
\hline
\end{tabular}

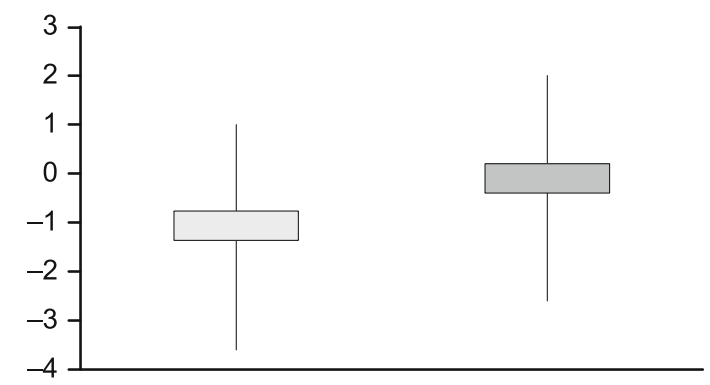

Fig. 8. Correction of error after teoretical calibration STN in $\mathrm{x}$ axis left side, box-avarage error, line standard deviaton in $\mathbf{m m}$.

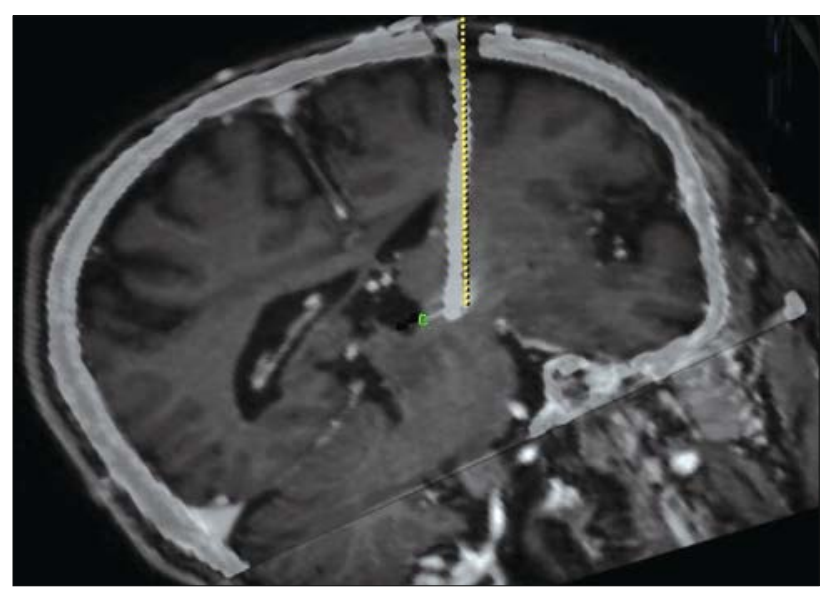

Fig. 9. Electrode bending in coronal plane on magnetic resonance image. Electrode is seen deviated to medial direction in StimLock and target area relative to planned trajectory.
After theoretical calibration, the average error in $\mathrm{x}$ axis on the left side decreased from $1.065 \mathrm{~mm}$ to medial to $0.1 \mathrm{~mm}$ to the medial direction (standard deviation 1.17). Potentially important error more than $2 \mathrm{~mm}$, which was present in 5 patients from 20 (25\%) after theoretical calibration droped to 1 case from 20 (5\%) (Fig. 8).

On the right side, theoretical calibration decreased the error of more than $2 \mathrm{~mm}$ in $\mathrm{x}$ axis from 1 patient from 20 patients (5\%) to $0 \%$.

In axis $\mathrm{y}$, there was no electrode exceeding $2 \mathrm{~mm}$ error, but in patients after implantation of electrode to STN on the left side, there was error close to $2 \mathrm{~mm}$ to the posterior direction in 3 electrodes, which was also corrected with the theoretical calibration.

The biggest error we recorded was in the electrode implanted to STN on the left side $3.6 \mathrm{~mm}$. This error required an operative reposition of the electrode, because of an insufficient stimulation effect. This error and the whole intracranial course of this electrode was analysed from its anchoring in StimLock burr hole cover to its end. Angulation and bending of the electrode was noticed intracranially. Similar bending was seen also in other patients, though it was most pronounced in this patient with the biggest error in the electrode position. The position of the electrode relative to the planned trajectory was analysed in other patients in the plane between the outer margins of the burr hole perpendicularly, what we considered the plane of securing mechanism of support clip of StimLock. Bending of electrodes was evaluated in the mediolateral and anteroposterior direction (Fig. 9).

We analysed the group of 20 patients with the implantation of electrode to left STN and according to the error and bending of electrode intracranially they were divided to 3 groups. In the first group of 6 patients $(30 \%$ ), the error of less than $0.9 \mathrm{~mm}$ in $\mathrm{x}$ axis was observed and the position of definitive electrode in the plane of StimLock was close to the planned trajectory and close to the centre of the burr hole (Fig. 10A, Tab. 2).

The second group of 9 patients ( $45 \%$ ) had the position of planned trajectory in the plane of StimLock lateral from its centre and the position of definitive electrode was moved to medial direction toward the centre of burr hole. In this group, the biggest error to the medial direction was observed in the target, most of them close to $2 \mathrm{~mm}$ ( 7 from 9 patients). All electrodes were bended with the convexity to contralateral lateral direction (Fig. 10B, Tab. 3).
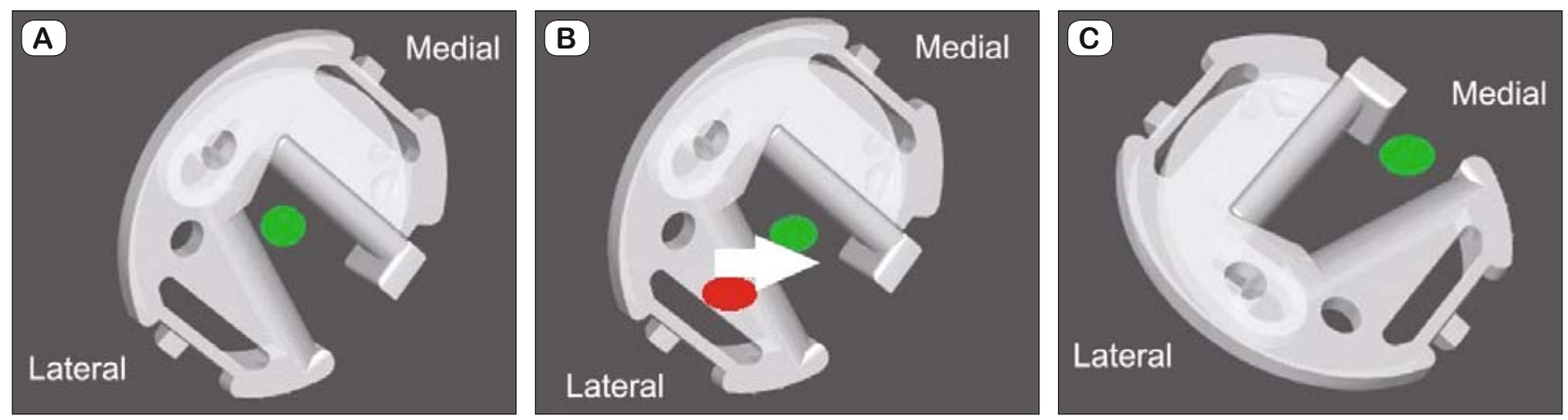

Fig. 10. Anchoring of electrode in StimLock burr hole cover. A - no shift of electrode during anchoring, B - shift of electrode during anchoring, $\mathrm{C}$ - anchoring of electrode close to the margin of the burr hole. 
Tab. 2. Group of patients with a position of definitive electrode in the plane of StimLock close to the planned trajectory and close to the centre of the burr hole.

\begin{tabular}{ccc}
\hline Patient & Error in $\mathrm{x}$ axis in the target & Electrode bending \\
\hline 1 & $0.8 \mathrm{~mm}$ medial & $0.5 \mathrm{~mm}$ \\
2 & $0.6 \mathrm{~mm}$ medial & $0.7 \mathrm{~mm}$ \\
3 & $0 \mathrm{~mm}$ & $0.7 \mathrm{~mm}$ \\
4 & $0.4 \mathrm{~mm}$ medial & $1.3 \mathrm{~mm}$ \\
5 & $0 \mathrm{~mm}$ & $0.5 \mathrm{~mm}$ \\
6 & $0.9 \mathrm{~mm}$ medial & $1 \mathrm{~mm}$ \\
\hline
\end{tabular}

Tab. 3. Group of patients with a shift of electrode in the plane of StimLock to medial direction toward the centre of burr hole.

\begin{tabular}{cccc}
\hline Patient & $\begin{array}{c}\text { Error in x axis } \\
\text { in the target }\end{array}$ & $\begin{array}{c}\text { Shift in StimLock } \\
\text { medialy }\end{array}$ & Electrode bending \\
\hline 1 & $2.4 \mathrm{~mm}$ medial & yes & $1.3 \mathrm{~mm}$ \\
2 & $1.9 \mathrm{~mm}$ medial & yes & $0.4 \mathrm{~mm}$ \\
3 & $3.6 \mathrm{~mm}$ medial & yes & $3 \mathrm{~mm}$ \\
4 & $2.1 \mathrm{~mm}$ medial & yes & $0.9 \mathrm{~mm}$ \\
5 & $2.3 \mathrm{~mm}$ medial & small shift yes & $1.5 \mathrm{~mm}$ \\
6 & $2.3 \mathrm{~mm}$ medial & yes & $1.2 \mathrm{~mm}$ \\
7 & $2 \mathrm{~mm}$ medial & yes & $1.2 \mathrm{~mm}$ \\
8 & $1.1 \mathrm{~mm}$ medial & yes & $1.5 \mathrm{~mm}$ \\
9 & $0.9 \mathrm{~mm}$ medial & yes & $1.2 \mathrm{~mm}$ \\
\hline
\end{tabular}

The third group of 5 patients $(25 \%)$ had the definitive electrode close to the margins of the burr hole and various errors in the target were present $(0.1 \mathrm{~mm}$ to lateral, $1.4 \mathrm{~mm}$ to medial, 0.1 $\mathrm{mm}$ to lateral, $0 \mathrm{~mm}, 0 \mathrm{~mm}$ ). There was a different anchoring of electrode in this group with rotating support clip (Fig. 10C).

On the right side, where smaller average error was observed laterally, it was likewise. The biggest error to the lateral direction was $2.2 \mathrm{~mm}$ and $2 \mathrm{~mm}$ and there was a shift of the definitive electrode to the lateral direction relative to planned trajectory and electrode was bended with medial convexity. On this side, there were more patients corresponding to the group with a small error in the plane of StimLock and target area.

\section{Discussion}

The use of more tracks in 3D mapping of the target in DBS allows an inaccuracy correction, but on the other side, brings a risk of haemorrhagic complications. Past studies comparing 3D mapping of the target and direct MR guided planning showed an inferior accuracy of MR guided planning (6). The progress in MR imaging, better protocols with a superior resolution, and elimination of distortion results in more centres inclining to MR guided planning, sometimes without a microelectrode recording and using one track $(7,8,9)$.

The incidence of haemorrhagic complications is found in literature in the review studies $(10,11)$ as well as in studies with a higher number of patients $(12,13,7,14)$. The average incidence of haemorrhagic complications is $5 \%$, asymptomatic in $1.9 \%$, symptomatic in $2.1 \%$ and resulting in permanent deficit or death in $1 \%$.

There are several studies analysing risk factors of haemorrhagic complications in DBS $(14,15)$.
The risk of haemorrhagic complication is higher when using MER, which was confirmed by studies $(10,8)$, where there was a significantly higher incidence of haemorrhage in centres using microelectrodes compared to the centres, which did not use them. Other studies found 4-5 times higher risk of haemorrhage with using MER (16). The number of penetrations correlated with haemorrhage, in the group of patients without haemorrhagic complication, the average number of penetrations was $2.9 \pm 1.8$ compared to the group of patients with haemorrhage and the average number of $4.1 \pm 2.0$ penetrations. Some studies didn't show a significant difference comparing the number of penetrations, but in the two groups there was a trend with a higher number of penetrations in the group with haemorrhage $(15,14)$. Some studies showed no difference in complications with the use of MER (17).

This was the rationale for our effort to decrease the number of penetrations.

There are several studies dealing with the accuracy of different stereotactic systems. Review study (18)compared the accuracy in frame-based, frameless (19) and iMR guided systems.

Some of them measured the targeting inaccuracy without analysing its direction or causes. Holl et al analysed error in 165 patients, where there was a significant difference between left and right side, error was higher on left side and when the angle of trajectory in coronal plane was more than $15^{\circ}$. The vector error in this study was bilateral $0.7 \mathrm{~mm}$ to posterior in y axis, in $\mathrm{x}$ axis it was error to the medial direction $1.1 \mathrm{~mm}$ on the left side and 0.1 $\mathrm{mm}$ to lateral on right side (20).

Daniluk et al observed the error in the position of electrode after technical errors were excluded. Bilateral error in posteromedial direction was described, which was reduced by compensation anterolaterally during a trajectory planning. There was no difference between brain hemispheres. Bending of electrode was described, and as the cause of this bending was not specified „brain related“" factor was expected (21).

Individual steps and potential errors of stereotactic framebased deep brain stimulation operation are:

- Fixation of stereotactic frame

- CT/MR imaging (technical error, image fusion error)

- Registration, planning (technical error, human error)

- Burr hole (brain shift, liquorrhoea, pneumocephalus)

- Implantation and fixation of electrode (mechanical error, human error, electrode migration)

The analysis of individual steps of the operation is essential to exclude a technical error. Accumulation of small errors in different steps can lead to a significant error with suboptimal stimulation effect. Some of these errors are random regarding the direction (imaging, image fusion, registration).

Brain related factors, which can influence the accuracy of electrode position are the brain shift, intraoperative liquorrhoea and pneumocephalus (22).

In pneumocephalus, as the result of intraoperative liquorrhoea, posterior shift in y axis is expected. In our patients, only the average posterior shift corrected by calibration was observed in STN electrode implantation on the left side. There were several patients with the error close to $2 \mathrm{~mm}$, where on postoperative CT there was 
pneumocephalus with the biggest thickness in the group. However, there were patients in the Gpi group, who had similar thickness of pneumocephalus, without the shift of electrode posteriorly. We used a guiding tube in place in another track used for 3D mapping during the whole electrode implantation, which hold brain tissue and prevented brain shift.

Generally, it is important to prevent pneumocephalus and brain shift during operation, which is described in several studies (23, 24). Brain shift is observed in y axis ranging from $0.4-2.2 \mathrm{~mm}$, shift in $\mathrm{x}$ axis is close to 025 . Another mechanism of brain shift described is the pressure of electrode during implantation.

Important factor in pneumocephalus prevention is the position of the head during the operation.

Head in semiseated position can decrease a leak of cerebrospinal fluid by means of gravity, but it is different from position during imaging, which can lead to brain shift. Some studies describe the maximal brain shift subcorticaly to $5.7 \mathrm{~mm}$ (23). According to the study (24), optimal position of the head is supine with slightly elevated head in $15^{\circ}$, which minimizes a negative intracranial pressure.

We analysed the trajectory of the electrode relative to planned trajectory and the process of implantation and anchoring of electrode was found to be related to electrode bending intracranially, which was in correlation with the error in the target area.

Several studies described the electrode movement in different anchoring devices (18), when using StimLock it was in the average $1.8 \mathrm{~mm}$. Other study compared movement of electrode using StimLock and anchoring electrode with microplate where movement was $1.5 \pm 0.6 \mathrm{~mm}$ and $2.3 \pm 2 \mathrm{~mm}$ respectively (26). Zibly described movement and electrode bending in the target area seen on intraoperative x-ray after anchoring of electrode in StimLock, after releasing anchoring cover electrode returned to the original position (27).

The recommendation for the electrode implantation and anchoring in StimLock burr hole cover is using the standard burr hole diameter, insertion of the base of StimLock to the burr hole and fixation with screws, there is one exit slot for electrode in the base, orientation of exit slot should be in the direction of tunnelling toward the implanted pulse generator (IPG), insertion of support clip to the base around electrode, orientation of the support clip so that the static side of securing mechanism is against the lead body facing away to the exit slot, avoiding significant contact between the lead body and the support clip is recommended, lead stylet in place and gentle rotation of the securing mechanism of the support clip until it snaps into the locked position against the lead, removal of electrode stylet and leading electrode to exit slot (Fig. 11).

According to the recommendations, the support clip should be inserted to the base always in the same orientation, so that its static part is oriented to the part of base with exit slot. The optimal position of the electrode in the support clip is reduced to a small area close to the centre of burr hole. There is often not enough attention paid to the position of electrode in burr hole, especially when considering that the entry of electrode is not vertical to the skull and the position of more tracks can be used for a definitive electrode placement. At the time of anchoring of electrode, there is a stylet inside which makes the electrode more firm and stylet

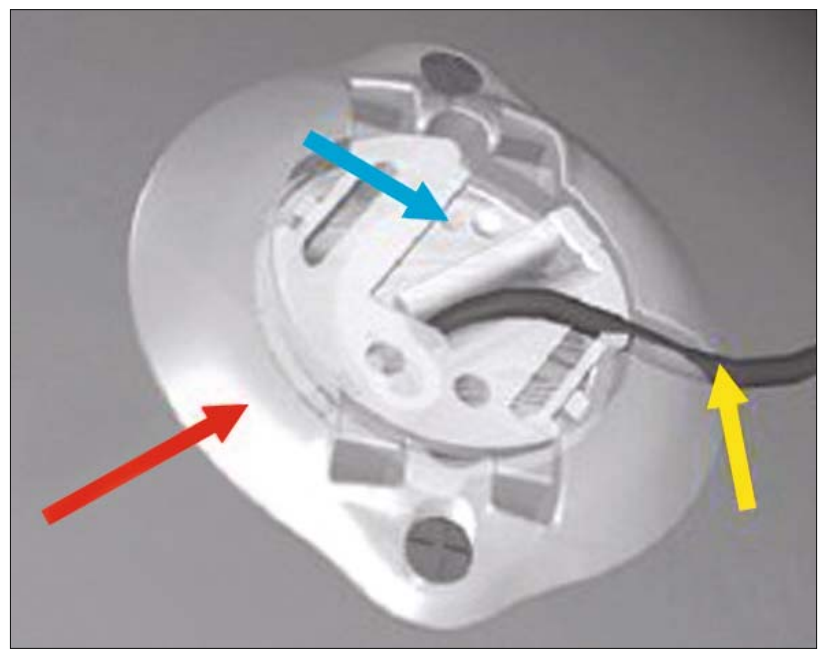

Fig. 11. StimLock burr hole cover with anchored electrode, yellow arrow - electrode in exit slot, red arrow - base of StimLock, blue arrow- securing mechanism of the support clip.

is removed after anchoring. Shift sensitive direction in this case is the mediolateral moving electrode toward the centre of the burr hole. Other traction can be present because of the oblique entry of the electrode to the plane of StimLock.

There are different angles of entry in STN and Gpi, because Gpi is located more laterally, the entry is in a more acute angle. So is the entry to the cerebral parenchyma, which can possibly prevent a greater electrode bending. This is a possible cause why there is less error in smaller angles in coronal plane typical for Gpi targeting. Patients with Gpi targeting had electrode entry angle to medial plane in average $5.05^{\circ}\left(0.2-9.5^{\circ}\right)$ and patients with electrode implantation to STN had the entry angle to medial plane in average $22.3^{\circ}\left(15.1-27.7^{\circ}\right)$.

The calibration of stereotactic coordinates can decrease the average error, but because it doesn't analyse causes of prevalent error in individual positions of electrodes, it can lead to a shift to the contralateral direction away from the target.

More attention should be paid to the anchoring electrode. If the position of trajectory is not in the middle in StimLock, it is possible to enlarge burr hole in mediolateral direction to correct it before anchoring of StimLock base. When using more penetrations, the problem is that in the beginning it is not known, which penetration will be used for the definitive electrode implantation. The fixation of base of StimLock could be done at the time of electrode anchoring and the orientation of exit slot by rotating base of StimLock could be adjusted according to the position of the definitive electrode.

The change in the construction of StimLock could be helpful, to enable more freedom for placing the support clip and adjust it to the position of trajectory in the burr hole, there could be more than one exit slot around the base of StimLock, so the static part of the support clip could be still oriented towards exit slot, but with a possibility to choose more exit directions.

Inserting some sponge absorbable material to the medial and lateral sides of electrode in the burr hole bellow support clip could also prevent an electrode bending. 
The standard intraoperative control of electrode position is $\mathrm{X}$-ray $\mathrm{C}$ arm with imaging from the side of the head, where it is not possible to see shift in mediolateral direction. In the case of the biggest error in our patient $3.6 \mathrm{~mm}$ medially, there was just $0.3 \mathrm{~mm}$ antero-posterior error, which could be seen on the control $\mathrm{X}$ - ray. A control also in anteroposterior plane or using $\mathrm{O}$ arm (28, 29) could detect this error.

\section{Conclusion}

A substantial shift of electrode is possible in anchoring devices. This can cause an error of targeting to prevalent direction. A traction of electrode in this device can cause bending of electrode intracranially, resulting in the shift of electrode in the target area. More attention should be paid to anchoring electrode and different measures can be used during anchoring the electrode to avoid this shift.

\section{References}

1. Anheim M, Batir A, Fraix V, Benabid AL Pollak P. Improvement in Parkinson Disease by subthalamic nucleus stimulation based on electrode placement: effects of reimplantation. Arch Neurol 2008; 65: 612-616.

2. Ellis TM, Foote KD, Fernandez HH et al. Reoperation for suboptimal outcomes after deep brain stimulation surgery. Neurosurgery 2008; 63 (4): 754-760.

3. Richardson RM, Ostrem JL, Starr PA. Surgical repositioning of misplaced subthalamic electrodes in Parkinson's disease: location of effective and ineffective leads. Stereotact Funct Neurosurg 2009; 87 (5): 297-303.

4. Benetin J, Valkovič $\mathbf{P}$ et al. Parkinsonova choroba. Bratislava: Herba, 2009.

5. Bot M, Van den Munckhof P, Bakay R, Stebbins G, Verhagen Metmana L. Accuracy of Intraoperative Computed Tomography during Deep Brain Stimulation Procedures: Comparison with Postoperative Magnetic Resonance Imaging. Stereotact Funct Neurosurg 2017; 95 (3): 183-188.

6. Zonenshayn M, Rezai AR, Mogilner AY, Beric A, Sterio D, Kelly PJ. Comparison of anatomic and neurophysiological methods for subthalamic nucleus targeting. Neurosurgery 2000; 47 (2): 282-292.

7. Hirabayashi H, Tengvar M, Hariz M. Stereotactic Imaging of the pallidal target. Movement Disorders 2002; 17 (Suppl 3): S130-134.

8. Patel NK, Plaha P, Gill SS. Magnetic resonance imaging-directed method for functional neurosurgery using implantable guide tubes. Neurosurgery 2007; 61 (Suppl 2): 358-365.

9. Zrinzo L, Foltynie T, Limousin P, Hariz MI. Reducing hemorrhagic complications in functional neurosurgery: a large case series and systematic literature review. J Neurosurg 2012; 116 (1): 84-94.

10. Alkhani A, Lozano AM. Pallidotomy for parkinson disease: a review of contemporary literature. J Neurosurg 2001; 94 (1): 43-49.

11. Hamani C, Richter E, Schwalb JM, Lozano AM. Bilateral subthalamic nucleus stimulation for Parkinson's disease: a systematic review of the clinical literature. Neurosurgery 2005; 56 (6): 1313-1321.

12. Deep-Brain Stimulation for Parkinson's Disease Study Group. Deep-brain stimulation of the subthalamic nucleus or the pars interna of the globus pallidus in Parkinson's disease. N Engl J Med 2001; 345 (13): 956-963.
13. Deuschl G, Schade-Brittinger C, Krack P et al. German Parkinson Study Group, Neurostimulation Section. A randomized trial of deep-brain stimulation for Parkinson's disease. N Engl J Med 2006; 355 (9): 896-908.

14. Xiaowu H, Xiufeng J, Xiaoping $Z$ et al. Risks of intracranial hemorrhage in patients with Parkinson's disease receiving deep brain stimulation and ablation. Parkinsonism Relat Disord 2010; 16 (2): 96-100.

15. Elias WJ, Sansur CA, Frysinger RC. Sulcal and ventricular trajectories in stereotactic surgery. J Neurosurg 2009; 110 (2): 201-207.

16. Palur RS, Berk C, Schulzer M, Honey CR. A metaanalysis comparing the results of pallidotomy performed using microelectrode recording or macroelectrode stimulation. J Neurosurg 2002; 96 (6): 1058-1062.

17. Reddy S, Fenoy A, Furr-Stimming E, Schiess M, Mehanna R. Does the Use of Intraoperative Microelectrode Recording Influence the Final Location of Lead Implants in the Ventral Intermediate Nucleus for Deep Brain Stimulation? Cerebellum 2017; 16 (2): 421-426.

18. Li Z, Zhang J, Ye Y, Li X. Review on Factors Affecting Targeting Accuracy of Deep Brain Stimulation Electrode Implantation between 2001 and 2015. Stereotact Funct Neurosurg 2016; 94: 351-362.

19. Krahulík D, Nevrlý M, Otruba P et al. Placement Accuracy of Deep Brain Stimulation Electrodes using the NexFrame Frameless System. Cesk Slov Neurol N 2017; 80/113 (2): 208-212.

20. Holl EM, Petersen EA, Foltynie T, Martinez-Torres I, Limousin P, Hariz MI, Zrinzo L. Improving targeting in image-guided frame-based deep brain stimulation. Neurosurgery 2010; 67 (Suppl 2): 437-447.

21. Daniluk S, Davies KG, Novak P, Vu T, Nazzaro JM, Ellias SA. Isolation of the brain-related factor of the error between intended and achieved position of deep brain stimulation electrodes implanted into the subthalamic nucleus for the treatment of Parkinson's disease. Neurosurgery 2009; 64 (Suppl 2): 374-382.

22. Bentley JN, Guan Z, Cummings KS, Chou KL, Patil PG. Influence of Intracranial Air on Electrode Position and Clinical Outcomes following Deep Brain Stimulation for Parkinson's Disease. Stereotact Funct Neurosurg 2017; 95 (1): 6-12.

23. Elias WJ, Fu KM, Frysinger RC. Cortical and subcortical brain shift during stereotactic procedures. J Neurosurg 2007; 107 (5): 983-988.

24. Miyagi Y. Supine positionfor DBS surgery: how to prevent a brain shift? Funct Neurosurg 2013; 52: 14-18.

25. Petersen EA, Holl EM, Martinez-Torres I, Foltynie T, Limousin P, Hariz MI, Zrinzo L. Minimizing brain shift in stereotactic functional neurosurgery. Neurosurgery 2010; 67 (Suppl 3): 213-221.

26. Contarino MF, Bot M, Speelman JD et al. Postoperative displacement of deep brain stimulation electrodes related to lead-anchoring technique. Neurosurg 2013; 73 (4): 681-688.

27. Zibly Z, Sharma M, Shaw A, Yeremeyeva E, Deogaonkar M, Rezai A. Deep brain stimulation (DBS), lead migration, and the stimloc cap: Complication avoidance. Neurol India 2014; 62: 703-704.

28. Carlson JD, McLeod KE, McLeod PS, Mark JB. Stereotactic Accuracy and Surgical Utility of the O-Arm in Deep Brain Stimulation Surgery. Oper Neurosurg 2017; 13 (1): 96-107.

29. Caire FA, Gantois CA, Torny FB, Ranoux DB, Maubon AC, Moreau JJ. Intraoperative Use of the Medtronic O-arm for Deep Brain Stimulation Procedures Stereotact Funct Neurosurg 2010; 88: 109-114.

Received May 21, 2017. Accepted July 14, 2017. 